\title{
Exposition à l'arsenic et diabète de type 2 : résultats de l'Enquête canadienne sur les mesures de la santé de 2007 à 2009
}

\author{
S. K. Feseke M.D. (1, 2); J. St-Laurent, Ph. D. (1); E. Anassour-Sidi, M. Sc. (1); P. Ayotte, Ph. D. (1, 2, 3); \\ M. Bouchard, Ph. D. (4); P. Levallois, M.D. (1, 2, 3)
}

Cet article a fait l'objet d'une évaluation par les pairs.

Diffuser cet article sur Twitter

\section{Résumé}

Introduction : L'arsenic inorganique et ses métabolites sont considérés comme dangereux pour la santé humaine. Même si plusieurs études ont indiqué des associations entre une faible exposition à l'arsenic et le diabète sucré aux États-Unis et au Mexique, ces associations n’ont pas été étudiées chez les Canadiens. Nous avons analysé l'association entre l'exposition à l'arsenic, mesurée par la concentration d'arsenic total dans l'urine, et la prévalence du diabète de type 2 (DT2) chez 3151 participants adultes du cycle 1 (2007 à 2009) de l'Enquête canadienne sur les mesures de la santé (ECMS).

Méthodologie : La glycémie et l'hémoglobine glycosylée ont été mesurées chez les participants. L'arsenic total a été dosé par une analyse d'urine. De plus, les participants ont répondu à un questionnaire détaillé sur leurs habitudes de vie et leurs antécédents médicaux. Nous avons analysé l'association de la concentration urinaire d'arsenic avec le DT2 et le prédiabète à l'aide d'une régression logistique multivariée ajustée pour les facteurs de confusion potentiels.

Résultats : La concentration urinaire d'arsenic total était positivement associée à la prévalence de DT2 et de prédiabète : les rapports de cotes ajustés étaient respectivement de 1,81 (IC à $95 \%: 1,12$ à 2,95 ) et de 2,04 (IC à $95 \%: 1,03$ à 4,05) lorsqu'on comparait les concentrations urinaires d'arsenic les plus élevées (quatrième quartile) aux plus faibles (premier quartile). L'arsenic total urinaire était également associé au taux d’hémoglobine glycosylée chez les diabétiques non traités.

Conclusion : Nous avons noté des associations significatives entre l'exposition à l'arsenic et la prévalence de DT2 et de prédiabète dans la population canadienne. L'inférence causale est limitée car il s'agit d'une étude transversale exempte d'évaluation de l'exposition à long terme.

Mots-clés : arsenic urinaire, Enquête canadienne sur les mesures de la santé, diabète de type 2, enquête auprès de la population

\section{Introduction}

D’après la Loi canadienne sur la protection de l'environnement, l'arsenic inorganique et ses métabolites sont assez toxiques pour « constituer un danger au Canada pour la vie ou la santé humaines " ${ }^{1}$. En fait, l'arsenic, présent sous forme organique et
Principales constatations

- Notre étude portait sur 1520 hommes et 1631 femmes âgés de 20 à 79 ans dont on connaissait la concentration d'arsenic dans l'urine. Les critères de définition du diabète étaient la glycémie à jeun égale ou supérieure à $126 \mathrm{mg} / \mathrm{dl}$, l'hémoglobine A1c (HbA1c) égale ou supérieure à 6,5\% ou un traitement contre le diabète.

- La concentration urinaire d'arsenic total était positivement associée à la prévalence du diabète de type 2 et du prédiabète : les rapports de cotes ajustés étaient de 1,81 (IC à $95 \%$ : 1,12 à 2,95 ) et de 2,04 (IC à $95 \%$ : 1,03 à 4,05) respectivement lorsqu'on comparait les concentrations urinaires d'arsenic les plus élevées (quatrième quartile) avec les plus faibles (premier quartile).

- L'arsenic total urinaire était également associé à la concentration d'hémoglobine glycosylée chez les diabétiques non traités.

inorganique et provenant principalement de sources naturelles, est l'un des éléments les plus toxiques de l'environnement. Les Canadiens sont exposés à l'arsenic principalement par les aliments et par l'eau potable, le sol et l'air ambiant. Même si la concentration d'arsenic dans l'eau potable de la plupart des municipalités canadiennes est inférieure à la ligne directrice de Santé Canada, soit $10 \mu \mathrm{g} / \mathrm{l}^{2}$,

Rattachement des auteurs :

1. Axe santé des populations et pratiques optimales en santé, Centre de recherche du CHU de Québec, Québec (Québec), Canada

2. Département de médecine sociale et préventive, Faculté de médecine, Université Laval, Québec (Québec), Canada

3. Direction de la santé environnementale et de la toxicologie, Institut national de santé publique du Québec, Québec (Québec), Canada

4. Département de santé environnementale et santé au travail, Chaire d'analyse et de gestion des risques toxicologiques, École de santé publique, Université de Montréal, Montréal (Québec), Canada

Correspondance: Solange Keboya Feseke, Axe santé des populations et pratiques optimales en santé, Centre de recherche du CHU de Québec, Québec (Québec) G1V 1S6; tél. : 418-653-4313; courriel : fesekekeboya@yahoo.fr 
elle y est supérieure dans certaines zones de plusieurs provinces (particulièrement celles desservies par des puits privés) ${ }^{2}$.

Les produits de la mer représentent la source alimentaire d'arsenic organique la plus importante $\mathrm{e}^{3,4}$. Le principal composé arsenical organique présent dans la majorité des aliments d'origine marine est l'arsénobétaïne, considérée sans danger ${ }^{5}$. L'arsenic inorganique, la forme la plus toxique du métalloïde ${ }^{6}$, est métabolisé dans le foie et transformé en dérivés monométhylés et diméthylés qui sont excrétés dans l'urine tout comme l'arsenic inorganique non métabolisé ${ }^{6,7}$. Le sélénium peut influencer la toxicité de l'arsenic ${ }^{8}$.

Une exposition même faible à l'arsenic inorganique augmente le risque de lésions cutanées précancéreuses ${ }^{9,10}$, d’hyperten$\operatorname{sion}^{11,12}$ et de troubles neurologiques ${ }^{13}$. Des études d'observation chez l'humain et des études expérimentales chez les animaux ont montré que l'arsenic est potentiellement diabétogénique ${ }^{14}$. Cet effet de l'arsenic sur le diabète de type 2 (DT2), une maladie qui touche environ 346 millions de personnes dans le monde ${ }^{15,16}$ et 2,4 millions de personnes au Canada ${ }^{17}$, constitue un enjeu majeur en santé publique ${ }^{14,18}$.

Les premières études ont été menées au sein de populations exposées à de fortes concentrations d'arsenic dans l'eau potable à Taïwan et au Bangladesh, ainsi que dans le cadre d'études sur la santé au travail chez des ouvriers de fonderies de cuivre et des ouvriers du verre aux États-Unis et en Europe. Les méthodes de mesure de l'exposition diffèrent selon les études : elles vont de l'estimation de l'exposition pour une région en fonction du dosage de l'arsenic dans l'eau potable à l'estimation de l'exposition individuelle fondée sur des données détaillées concernant la consommation d'eau, les antécédents professionnels ou certains biomarqueurs de l'exposition. Une recension systématique des études épidémiologiques sur l'exposition à l'arsenic et le DT2 a indiqué que la majorité de ces études employaient des méthodes dites écologiques pour évaluer l'exposition et qu'elles n'ont pas été ajustées pour les facteurs de confusion potentiels ${ }^{14}$. Certaines études ayant utilisé la concentration urinaire d'arsenic comme biomarqueur de l'exposition n'ont pas décelé d'association entre l'exposition à l'arsenic et le diabète ${ }^{19,20}$, alors que d'autres ont signalé une relation dose-réponse $e^{21-27}$. Par ailleurs, aucune étude n'a évalué cette association dans la population canadienne. Dans ce cadre, cette étude visait donc principalement à évaluer l'association entre l'exposition à l'arsenic, mesurée à l'aide de la concentration d'arsenic total dans l'urine, et la prévalence de DT2 chez les participants adultes du premier cycle de l'Enquête canadienne sur les mesures de la santé (ECMS).

\section{Méthodologie}

\section{Population étudiée}

Nous avons eu recours aux données transversales du cycle 1 de l'ECMS, une enquête à échantillonnage complexe conçue pour recueillir des données auprès un échantillon représentatif d'environ 5600 Canadiens âgés de 6 à 79 ans entre 2007 et 2009. L'ECMS couvre environ 96,3\% de la population canadienne habitant des logements privés dans l'ensemble des provinces et des territoires. Toutefois, les personnes vivant en établissement, les membres à temps plein des Forces canadiennes ainsi que les personnes vivant dans les réserves et certaines régions éloignées en sont exclues et nous avons exclu également les participants âgés de moins de 20 ans. Nous avons donc pour cette étude les données sur 3517 participants âgés de 20 à 79 ans.

\section{Collecte des données}

Les données ont été recueillies entre mars 2007 et février 2009 dans 16 sites situés dans les provinces de l'Atlantique (Moncton, Nouveau-Brunswick), au Québec (Québec, Montréal, Montérégie, sud de la Mauricie), en Ontario (Charlington, North York, Don Valley, St. Catharines, Kitchener, comté de Northumberland), dans les Prairies (Edmonton et Red Deer, Alberta) et en Colombie-Britannique (Vancouver, Williams Lake et Quesnel) ${ }^{28}$. L'enquête comprenait une entrevue en personne à domicile suivie, de deux jours à six semaines plus tard, d'un examen physique et du prélèvement d'échantillons biologiques dans un centre d'examen mobile. Au total, le taux de réponse combiné du cycle 1 de l'ECMS était de $51,7 \%{ }^{29}$.

\section{Critères d'exclusion}

Dans le cadre de cette étude, les critères d'exclusion suivants ont été ajoutés : diabète de type 1 ( $\mathrm{n}=19)$, grossesse $(\mathrm{n}=11)$ et troubles hépatiques $(\mathrm{n}=72)$. Ce dernier critère a été retenu parce que les taux élevés d'enzymes hépatiques, même dans l'intervalle normal défini en pratique clinique, sont associés à un risque accru de diabète $^{30}$. Nous avons également exclu les participants qui ont signalé une consommation importante de fruits de mer (104 fois ou plus par année) ou une consommation importante de poisson (156 fois ou plus par année) ( $n=264)$ en se fondant sur la distribution du nombre de repas par semaine où des produits de la mer étaient consommés car ces participants étaient susceptibles de présenter une forte concentration d'arsenic dérivé d'aliments d'origine marine. Notre analyse finale a ainsi porté sur 3151 participants âgés de 20 à 79 ans.

\section{Analyse de l'arsenic urinaire}

\section{Prélèvement des échantillons d'urine}

Des échantillons d'urine ponctuels prélevés en milieu de jet $(60 \mathrm{ml})$ ont été fournis par les participants dans les centres d'examen mobiles. Les échantillons d'urine destinés à l'analyse de l'arsenic ont été recueillis dans des contenants exempts d'arsenic, expédiés sur glace sèche et entreposés à $-20^{\circ} \mathrm{C}$.

\section{Analyse des échantillons d'urine}

L'arsenic total a été dosé au Laboratoire de toxicologie de l'Institut national de santé publique du Québec conformément à un protocole normalisé accrédité en vertu de la norme ISO 17025 et soumis à de nombreux programmes internes et externes de contrôle de la qualité ${ }^{31}$. Les échantillons d'urine ont été dilués avec une solution d'acide nitrique aqueuse $(0,5 \%)$. On y a analysé l'arsenic total par la spectrométrie de masse à source plasma à couplage inductif (ICP-MS) à l'aide de l'appareil Elan DRC II. L'étalonnage apparié à la matrice a été effectué avec de l'urine de sujets non exposés ${ }^{32}$. Les concentrations urinaires ont 
également été ajustées pour les concentrations de créatinine, déterminées à l'aide de la méthode de Jaffé, afin de tenir compte de la dilution de l'urine ${ }^{33}$. Le seuil de détection de l'arsenic total urinaire était de $0,524 \mu \mathrm{g} / \mathrm{l}$. Le pourcentage de participants de l'étude dont la concentration urinaire d'arsenic total était inférieure au seuil de détection était de 0,35\%.

\section{Paramètres du diabète de type 2}

Les critères de définition du DT2 étaient une glycémie à jeun supérieure ou égale à 126 $\mathrm{mg} / \mathrm{dl}$ (7 mmol/l) ou une hémoglobine glycosylée $(\mathrm{HbA1c})$ supérieure ou égale à $6,5 \%$, conformément aux recommandations de l'Organisation mondiale de la Santé (OMS) et de l'American Diabetes Association $(\mathrm{ADA})^{34,35}$. L'autodéclaration soit d'un diagnostic de diabète posé par un médecin, soit de la prise d'insuline ou d'un hypoglycémiant oral a également servi de critère. Les critères de définition du prédiabète étaient une glycémie à jeun entre 100 et $125 \mathrm{mg} / \mathrm{dl}$ (5,6 à $6,9 \mathrm{mmol} / \mathrm{l})$ ou une HbA1c entre 5,7\% et $6,4 \%$ (conformément aux recommandations de l'OMS et de l'ADA) $)^{34,35}$.

\section{Glycémie à jeun}

Les échantillons de sang à jeun ont été prélevés chez 1714 participants de l'étude le matin après une période de jeûne d'au moins 10 heures. La glycémie du plasma veineux a été mesurée à l'aide du système de chimie clinique VITROS 5.1 FS d'OrthoClinical Diagnostics ${ }^{36}$.

\section{Concentration d'hémoglobine golycosylée}

La concentration d'HbA1c a été mesurée à l'aide du système de chimie clinique VITROS 5.1 FS d'Ortho-Clinical Diagnostics ${ }^{37}$.

\section{Autres paramètres de laboratoire}

La créatinine urinaire a été dosée à l'aide de la méthode colorimétrique de Jaffé afin de tenir compte de la dilution de l'urine des échantillons ponctuels. L'absorbance a été lue à $505 \mathrm{~nm}$ sur un autoanalyseur chimique Hitachi 917 (C-530) ${ }^{38}$.

La concentration urinaire de sélénium a été mesurée par ICP-MS en même temps que l'arsenic (analyse décrite ci-dessus). Le seuil de détection était de 0,08 $\mu \mathrm{mol} / \mathrm{l}$.

\section{Autres variables}

La pression artérielle a été mesurée électroniquement à l'aide d'un appareil oscillométrique automatique (BpTRU $\left.{ }^{\mathrm{MC}}\right)^{39}$. Nous avons adopté la définition de l'hypertension $\mathrm{du}$ Seventh Report of the joint national committee on prevention, detection, evaluation and treatment of high blood pressure, soit une pression systolique égale ou supérieure à $140 \mathrm{mmHg}$ et une pression diastolique égale ou supérieure à $90 \mathrm{mmHg}$. La prise d'antihypertenseurs ou l'autodéclaration d'un diagnostic d'hypertension posé par un médecin ont également servi de critères.

\section{Questionnaire}

Le questionnaire de l'ECMS a fourni des informations autodéclarées sur des variables sociodémographiques et de santé. Les tranches d'âge de l'ECMS étaient les suivantes : 20 à 39 ans, 40 à 59 ans et 60 à 79 ans. L'origine ethnique était classée en deux catégories : blanche et non blanche. Le niveau d'éducation était catégorisé de la manière suivante : pas de diplôme d'études secondaires, diplôme d'études secondaires, études postsecondaires partielles et diplôme d'études postsecondaires. Le tabagisme était réparti en trois catégories : fumeur, exfumeur et non-fumeur. La consommation d'alcool était répartie en trois catégories : actuelle, ancienne et jamais.

La fréquence totale de la consommation de poisson ou de fruits de mer a été répartie en quatre groupes en fonction de la consommation d'au moins un type de produits de la mer sur la liste de l'ECMS concernant l'alimentation : moins de 12 fois par année, 12 à 51 fois par année, 52 à 103 fois par année et 104 à 155 fois par année. Cette catégorisation était basée sur la conversion du nombre de repas par semaine où des aliments d'origine marine étaient consommés en nombre de repas par année dans la population étudiée.

L'indice de masse corporelle (IMC) a été calculé en divisant le poids (mesuré en kilogrammes) par la taille (mesurée en mètres) au carré.

Les participants ont indiqué s'ils consommaient de l'eau du robinet traitée par la municipalité, de l'eau d'un puits privé, de l'eau embouteillée ou de l'eau provenant d'une autre source d'eau potable. Ces réponses ont été classées en deux catégories : eau du robinet municipale ou autre.

\section{Analyse statistique}

Toutes les analyses statistiques ont été réalisées à l'aide de la version 9.3 du logiciel d'analyse statistique SAS (SAS Institute Inc., Cary, Caroline du Nord, États-Unis) et en appliquant les poids d'échantillonnage de l'ECMS. Nous avons estimé la variance (intervalle de confiance [IC] à 95\%) et effectué des tests d'hypothèse (chi carré) pour les différences entre les estimations à l'aide de la pondération bootstrap fournie avec les données afin de tenir compte de la conception complexe de l'échantillonnage ${ }^{40}$. Des méthodes de statistique descriptive (fréquences, moyennes géométriques) ont servi à estimer la concentration urinaire d'arsenic total en fonction des caractéristiques des participants. L'arsenic total urinaire, le sélénium, la glycémie plasmatique à jeun et l'HbA1c ont subi une transformation logarithmique aux fins de l'analyse de la moyenne géométrique. Les concentrations inférieures au seuil de détection par la méthode analytique ont été remplacées par une valeur égale à la moitié du seuil de détection ${ }^{42}$. Pour chaque variable de laboratoire, la moyenne géométrique des concentrations et l'IC à 95\% chez les participants atteints de prédiabète et de diabète ont été comparées aux valeurs obtenues chez les témoins non atteints, à l'aide de modèles de régression multivariés. La concentration urinaire d'arsenic total a été traitée soit comme une variable continue, soit en quartiles.

Nous avons eu recours à l'analyse de régression logistique binomiale (non diabétiques versus prédiabétiques ou diabétiques) et ordinale (les trois catégories simultanément) pour estimer les rapports de cotes (RC) et les intervalles de confiance à $95 \%$. Les modèles de régression logistique pour les paramètres portant sur la concentration urinaire d'arsenic total et sur le diabète ont été progressivement ajustés. Nous avons d'abord effectué un ajustement pour l'âge, le sexe, le niveau d'éducation, la consommation d'alcool, le tabagisme, l'IMC, l'hypertension et la 
créatinine urinaire pour refléter la dilution de l'urine des échantillons ponctuels ${ }^{43}$. Par la suite, chaque modèle a été ajusté pour la consommation de produits de la mer à l'aide des catégories présentées dans la section portant sur le questionnaire.

Nous avons analysé l'association entre la concentration urinaire d'arsenic et l'HbAlc dans les modèles stratifiés en fonction du statut lié au traitement du diabète, étant donné que l'HbAlc est un indicateur de la maîtrise du diabète ${ }^{44}$. Nous avons utilisé des modèles de régression logistique binomiale pour estimer les rapports de cotes de l'HbAlc en fonction de la concentration urinaire d'arsenic en appliquant la même stratégie d'ajustement que pour la première analyse sur le diabète. Nous avons analysé l'interaction du sélénium avec l'arsenic, car le sélénium peut protéger contre les effets toxiques de l'arsenic ${ }^{45}$.

Nous avons également utilisé les scores de propension pour évaluer les biais de sélection potentiels associés aux nonrépondants en équilibrant la distribution des covariables pour les principaux facteurs de risque ${ }^{46}$. Nous avons ajusté le modèle de régression pondéré sur le score de propension pour comparer la prévalence du DT2 et du prédiabète avec l'exposition à l'arsenic évaluée par l'analyse d'urine et pour étudier les prédicteurs possibles du DT2. Un modèle de régression pondéré sur le score de propension a ensuite servi à évaluer les associations de l'exposition à l'arsenic dosé dans l'urine chez les diabétiques non traités avec les résultats biologiques.

\section{Résultats}

\section{Caractéristiques des participants}

$\mathrm{Au}$ sein des 3151 participants (1520 hommes et 1631 femmes) de notre échantillon, la prévalence pondérée du DT2 et du prédiabète était de respectivement 7,1 \% (IC à $95 \%: 6,2 \%$ à 7,9\%) et 26,4\% (IC à $95 \%: 24,8 \%$ à $27,9 \%$ ). Les participants atteints de DT2 ou de prédiabète étaient significativement plus âgés, plus souvent de race non blanche, moins éduqués, et plus susceptibles d'avoir un IMC élevé que les témoins non atteints de prédiabète ou de DT2 (tableau 1). Les caractéristiques générales des prédiabétiques se situaient entre les caractéristiques des diabétiques et celles des témoins (tableau 1). La source d'approvisionnement en eau était la même pour les trois groupes.

La moyenne géométrique de la concentration urinaire d'arsenic total avait tendance à être plus élevée chez les femmes, les personnes âgées et les non-blancs ainsi que chez les consommateurs actuels d'alcool et les ex-fumeurs. Toutefois, les différences n'étaient pas statistiquement significatives (tableau 2).

\section{Arsenic et diabète de type 2}

La moyenne géométrique de la concentration urinaire d'arsenic total était plus

\section{TABLEAU 1}

État diabétique en fonction des caractéristiques des participants de l'étude, cycle 1 de l'ECMS, 2007 à 2009

Caractéristiques

État diabétique des participants (\%) (IC à 95\%)

$\begin{array}{ccc}\text { Ni diabétique, } & \text { Prédiabétique }^{\mathrm{b}} & \text { Diabétique de } \\ \text { ni prédiabétique } & (\mathrm{n}=831) & \text { type } 2^{\mathrm{C}} \\ (\mathrm{n}=2054) & & (\mathrm{n}=225)\end{array}$

\section{Âge (ans)}

20 à 39

40 à 59

60 à 79

Sexe

Femme

Homme

Niveau d'éducation

Études secondaires ou moins

Études postsecondaires partielles

Études universitaires

Origine ethnique

Blanche

Non blanche

Tabagisme

Fumeur

Ex-fumeur

Non fumeur

Consommation d'alcool

Actuelle
Ancienne
Jamais

IMC $\left(\mathrm{kg} / \mathrm{m}^{2}\right)$

Moins de 25

25 à 29

30 et plus

Source d'approvisionnement en eau

Eau du robinet municipale

Autre

$42,0(39,8$ à 42,8$)$
$35,5(34,5$ à 36,4$)$
$22,5(21,9$ à 23,6$)$

$18,7(17,6$ à 19,8$)$

$38,8(38,1$ à 39,5$)$

$42,5(41,9$ à 43,8$)$

$46,9(45,2$ à 47,8$)$

$53,1(51,4$ à 54,3$)$

$48,4(47,9$ à 49,7$)$

$51,6(49,2$ à 52,8)

$10,7(10,2$ à 11,8$)$

$25,4(24,9$ à 25,1$)$

$18,5(18,2$ à 18,9$)$

$24,3(23,1$ à 24,8$)$

$63,9(63,7$ à 64,6$)$

$57,2(56,4$ à 58,1$)$

$88,0(79,2$ à 88,7$)$

$12,0(11,2$ à 12,8$)$

$85,9(84,8$ à 86,8$)$

$14,1(13,2$ à 15,4$)$

21,6 (20,1 à 21,8)

$21,2(20,9$ à 21,7$)$

$29,3(28,7$ à 30,0$)$

$35,6(35,2$ à 36,3$)$

$49,1(48,5$ à 49,8$)$

$43,2(42,6$ à 43,8)

$88,2(87,5$ à 88,9$)$

$7,4(6,9$ à 7,8$)$

$79,7(78,8$ à 80,3$)$

$14,8(14,2$ à 16,1$)$

$4,4(4,0$ à 4,8$)$

$5,5(4,9$ à 5,8$)$

$42,1(41,6$ à 42,7)

$26,5(25,7$ à 27,2$)$

32,7 (31,8 à 33,0)

$31,5(31,1$ à 32,4$)$

$25,2(24,3$ à 25,8$)$

$42,0(41,2$ à 42,9$)$

$87,2(86,5$ à 87,8$)$

$85,9(85,2$ à 86,3$)$

$12,8(12,3$ à 13,6$)$
$8,9(8,4$ à 10,1$)$

27,6 (26,8 à 28,7)

$63,5(62,0$ à 64,8$)$

$55,1(54,2$ à 56,5$)$

$44,9(44,0$ à 45,8$)$

24,4 (23,9 à 24,8)

$25,3(24,2$ à 26,2$)$

50,3 (50,2 à 51,3)

$82,7(81,3$ à 83,1$)$

$17,3(16,2$ à 17,8$)$

$15,5(14,9$ à 16,1$)$

38,7 (38,2 à 39,4)

$45,8(45,3$ à 46,2$)$

$70,6(69,2$ à 79,9)

$20,6(19,9$ à 21,4$)$

$8,8(8,1$ à 9,2$)$

$15,3(14,4$ à 15,9$)$

26,6 (26,2 à 27,4)

$58,1(57,7$ à 60,2$)$

$83,3(82,9$ à 84,3$)$

$6,7(6,2$ à 7,1$)$

Abréviations : ECMS, Enquête canadienne sur les mesures de la santé; HbA1c, hémoglobine ǵlycosylée; IC, intervalle de confiance; IMC, indice de masse corporelle.

${ }^{a}$ Données manquantes : 41.

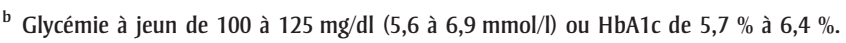

c Glycémie à jeun de $126 \mathrm{mg} / \mathrm{dl}$ ou plus (7 mmol// ou plus), HbA1c de 6,5\% ou plus ou autodéclaration de prise de médicaments ou de diagnostic de diabète par un professionnel de la santé. 
TABLEAU 2

Concentration urinaire d'arsenic en fonction des caractéristiques des participants du cycle 1 de I'ECMS, 2007 à 2009

\begin{tabular}{|c|c|c|c|}
\hline \multirow[t]{2}{*}{ Caractéristiques de la population } & \multirow[t]{2}{*}{ n (\%) } & \multicolumn{2}{|c|}{$\begin{array}{l}\text { Moyennes géométriques pour l'arsenic urinaire } \\
\qquad(\mu \mathrm{g} / \mathrm{I})(\mathrm{IC} \text { à } 95 \%)\end{array}$} \\
\hline & & $\begin{array}{l}\text { Arsenic urinaire non } \\
\text { corrigé pour tenir compte } \\
\text { de la créatinine }(\mu \mathrm{g} / \mathrm{l})\end{array}$ & $\begin{array}{l}\text { Arsenic urinaire corrigé } \\
\text { pour tenir compte de la } \\
\text { créatinine ( } \mu g \text { / } \\
\text { créatinine) }\end{array}$ \\
\hline \multicolumn{4}{|l|}{ Âge (ans) } \\
\hline 20 à 39 & $1059(33,6)$ & $11,4(10,0$ à 13,1$)$ & 12,8 (9,4 à 17,4) \\
\hline 40 à 59 & $1126(35,7)$ & $12,0(10,0$ à 14,3$)$ & $15,4(12,3$ à 19,2$)$ \\
\hline 60 à 79 & $966(30,7)$ & $11,4(9,3$ à 14,0$)$ & $16,0(11,8$ à 21,6$)$ \\
\hline \multicolumn{4}{|l|}{ Sexe } \\
\hline Femme & $1520(48,2)$ & 10,2 (7,6 à 13,7) & $16,4(12,5$ à 21,5$)$ \\
\hline Homme & $1631(51,8)$ & $13,2(10,0$ à 17,5$)$ & $12,8(9,6$ à 17,0$)$ \\
\hline \multicolumn{4}{|l|}{ Niveau d'éducation } \\
\hline Études secondaires ou moins & $429(13,6)$ & $11,2(9,2$ à 13,7$)$ & $13,7(10,6$ à 17,7$)$ \\
\hline Études postsecondaires partielles & $780(24,8)$ & $10,6(8,4$ à 13,2$)$ & $13,5(10,7$ à 16,9$)$ \\
\hline Études universitaires & $1942(61,6)$ & $14,1(10,2$ à 19,7$)$ & $17,1(12,8$ à 22,8$)$ \\
\hline \multicolumn{4}{|l|}{ Origine ethnique } \\
\hline Blanche & $2708(85,9)$ & $11,2(9,5$ à 13,2$)$ & $13,7(11,1$ à 16,9$)$ \\
\hline Non blanche & $443(14,1)$ & $14,0(9,6$ à 20,5$)$ & $18,4(12,0$ à 28,3$)$ \\
\hline \multicolumn{4}{|l|}{ Tabagisme } \\
\hline Fumeur & $655(20,8)$ & $10,5$ (8,3 à 13,2$)$ & $12,0$ (8,1 à 17,8$)$ \\
\hline Ex-fumeur & $990(31,4)$ & $12,6(10,0$ à 15,9$)$ & $15,5(12,0$ à 20,0$)$ \\
\hline Non fumeur & $1506(47,8)$ & $11,7(10,0$ à 13,6$)$ & $15,0(12,5$ à 18,1$)$ \\
\hline \multicolumn{4}{|l|}{ Consommation d'alcool } \\
\hline Actuelle & $2663(84,5)$ & $11,9$ (9,9 à 14,4$)$ & $14,5(11,5$ à 18,3$)$ \\
\hline Ancienne & $334(10,6)$ & $9,7$ (5,7 à 16,6$)$ & $13,9(10,9$ à 17,7$)$ \\
\hline Jamais & $154(4,9)$ & $11,3$ (8,2 à 15,6$)$ & $16,3(11,3$ à 23,5$)$ \\
\hline \multicolumn{4}{|l|}{ IMC $\left(\mathbf{k g} / \mathrm{m}^{2}\right)$} \\
\hline Moins de 25 & $1157(36,7)$ & $11,7(10,3$ à 13,3$)$ & $16,0(12,7$ à 20,1$)$ \\
\hline 25 à 29 & $989(31,4)$ & $12,1$ (9,9 à 14,7$)$ & $14,1(11,6$ à 17,0$)$ \\
\hline 30 et plus & $1005(31,9)$ & 11,2 (9,1 à 13,8) & 13,0 (9,8 à 17,4) \\
\hline \multicolumn{4}{|l|}{$\begin{array}{l}\text { Source d'approvisionnement en } \\
\text { eau }\end{array}$} \\
\hline Eau du robinet municipale & $2702(86,0)$ & $12,0(10,1$ à 14,2$)$ & $14,9(12,0$ à 18,6$)$ \\
\hline Autre & $449(14,0)$ & $10,0$ (5,9 à 16,9$)$ & 12,2 (6,8 à 21,9) \\
\hline
\end{tabular}

Abréviations : ECMS, Enquête canadienne sur les mesures de la santé; HbA1c, hémoglobine ǵlycosylée; IC, intervalle de confiance; IMC, indice de masse corporelle.

élevée chez les participants diabétiques $(12,9 \mu \mathrm{g} / \mathrm{l} ; \mathrm{IC}$ à $95 \%: 9,4$ à $17,7 \mu \mathrm{g} / \mathrm{l})$ et prédiabétiques $(12,5 \mu \mathrm{g} / \mathrm{l}$; IC à $95 \%: 10,1$ à $15,4 \mu \mathrm{g} / \mathrm{l})$ que chez les témoins $(11,5 \mu \mathrm{g} / \mathrm{l}$; IC à $95 \%: 9,4$ à $14,1 \mu \mathrm{g} / \mathrm{l})$. Après correction pour la créatinine urinaire, nous avons observé la même différence entre les participants atteints de prédiabète ou de diabète et les témoins (tableau 3). La concentration urinaire de sélénium ne différait pas significativement entre les trois groupes. les caractéristiques sociodémographiques (âge et sexe), les facteurs de risque de diabète, la créatinine urinaire et la consommation de produits de la mer $(\mathrm{RC}=1,8$; IC à $95 \%: 1,1$ à 3,0$)$. Par ailleurs, chez les participants prédiabétiques, une association semblable a été observée après ajustement pour les facteurs de confusion potentiels ( $\mathrm{RC}=2,1$; IC à $95 \%: 1,0$ à 4,1$)$.

La régression logistique ordinale se penchant sur le DT2, le prédiabète et le groupe témoin a révélé des résultats semblables, pour la concentration urinaire d'arsenic total et l'état diabétique, à ceux des modèles précédents portant uniquement sur le diabète ou le prédiabète. En outre, une tendance générale à l'augmentation des RC associée à la hausse de l'arsenic total urinaire et une relation dose-réponse statistiquement significative ont été notées (tableau 5).

Enfin, la concentration urinaire d'arsenic total n'était pas liée à l'HbAlc chez les diabétiques traités (tableau 6), mais elle était fortement associée à l'HbAlc chez les participants non traités, après ajustement pour les facteurs de confusion potentiels.

Dans cette étude, le sélénium n'a pas interagi avec les effets de l'arsenic (données non présentées).

Après pondération par probabilité inverse basée sur les scores de propension, nous avons noté que les résultats étaient semblables aux résultats obtenus à l'aide des premiers modèles de régression (données non présentées). Un modèle de régression réalisé pour évaluer l'association de l'exposition à l'arsenic mesurée dans l'urine chez les diabétiques non traités avec les résultats biologiques a révélé une association semblable (données non présentées).

à l'aide des modèles dérivés de l'analyse de régression logistique binomiale menée chez les participants atteints de DT2 et de prédiabète en fonction des quartiles de la concentration urinaire d'arsenic total. Les participants du quartile comprenant les plus fortes concentrations urinaires d'arsenic total présentaient un risque presque deux fois plus élevé de DT2 que les participants du quartile des plus faibles concentrations, après ajustement pour

\section{Analyse}

Nous avons relevé, dans un échantillon représentatif d'adultes canadiens ayant participé à l'ECMS de 2007 à 2009, une association positive entre la concentration urinaire d'arsenic total et la prévalence de DT2 et de prédiabète, après ajustement pour 
TABLEAU 3

Variables de laboratoire chez les participants de l'ECMS atteints de prédiabète ${ }^{\mathrm{a}}$ ou de diabète ${ }^{\mathrm{b}}$ ainsi que chez les témoins, cycle 1 de l'ECMS, 2007 à 2009

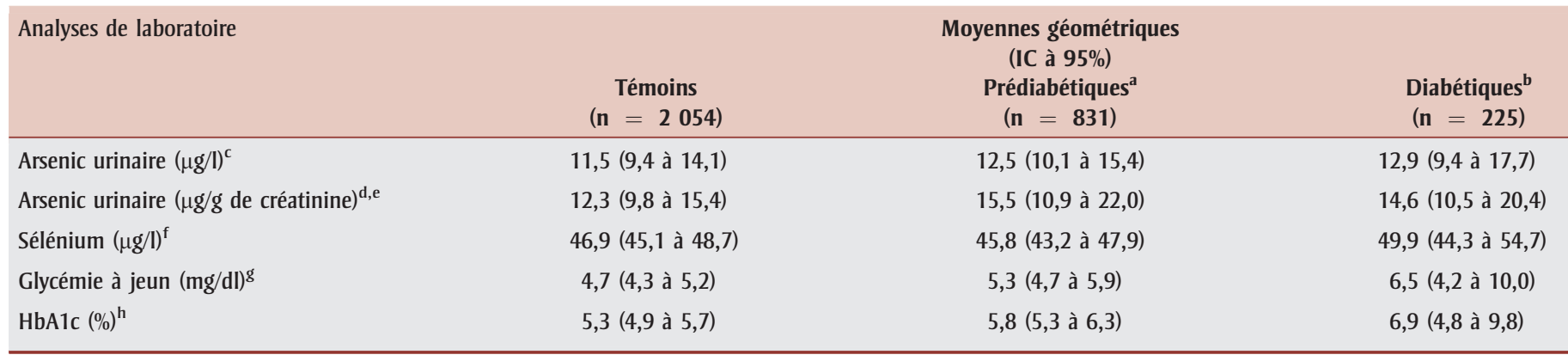

Abréviations : ECMS, Enquête canadienne sur les mesures de la santé; HbA1c, hémoglobine glycosylée; IC, intervalle de confiance.

${ }^{a}$ Glycémie à jeun : 100 à 125 mg/dl (5,6 à 6,9 mmol/l) ou HbA1c : 5,7 \% à 6,4 \%.

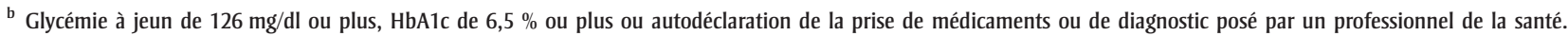

c Arsenic urinaire non corrigé pour tenir compte de la créatinine urinaire.

${ }^{\mathrm{d}}$ Arsenic urinaire corrigé pour tenir compte de la créatinine urinaire.

e Données manquantes pour l'arsenic urinaire corrigé afin de tenir compte de la créatinine urinaire : 39.

${ }^{f}$ Données manquantes pour le sélénium sont : 76.

${ }^{g}$ Données manquantes pour la glycémie à jeun : 1437.

h Données manquantes pour l'HbA1c : 106.

plusieurs facteurs de confusion potentiels et pour la consommation de produits de la mer. L'association entre l'arsenic et l'HbAlc était significative uniquement chez les participants diabétiques non traités.

Ces résultats sont similaires à ceux de plusieurs études antérieures portant sur de faibles expositions ainsi que d'études comportant des mesures précises des résultats et de l'exposition ${ }^{14,26}$. Ces dernières estimaient l'exposition à l'arsenic inorganique et à ses métabolites ${ }^{21,22,24}$ ou à l'arsenic inorganique par le dosage de l'arsenic total ajusté pour les marqueurs de la consommation de produits de la $\operatorname{mer}^{23,26}$. Nos résultats sont également conformes aux résultats de l'étude transversale ayant recours aux données du National Health Nutrition and Examination Survey (NHANES), qui évoquent une augmentation du risque de diabète en fonction de la concentration urinaire d'arsenic après ajustement pour l'arsenic provenant d'aliments d'origine marine ${ }^{23}$. Après ajustement pour les facteurs de risque de diabète et pour les marqueurs de consommation de produits de la mer, Navas-Acien et collab. ${ }^{23}$ ont noté que le RC du DT2 était de 2,6 (IC à 95\% : 1,1 à 6,0) lorsque les participants du $80^{\mathrm{e}}$ percentile de

TABLEAU 4

Analyse de régression logistique binomiale menée chez les participants atteints de prédiabète ${ }^{a}$ et de diabète de type $2^{b}$ ainsi que chez les témoins en fonction des quartiles de la concentration urinaire d'arsenic, cycle 1 de I'ECMS, 2007 à 2009

\begin{tabular}{|c|c|c|c|c|c|c|c|c|c|}
\hline \multirow{2}{*}{$\begin{array}{l}\text { Arsenic } \\
\text { urinaire } \\
(\mu g / l)^{c}\end{array}$} & \multicolumn{3}{|c|}{ Nombre de participants ${ }^{d}$} & \multicolumn{2}{|c|}{ RC brut (IC à 95\%) } & \multicolumn{2}{|c|}{$\begin{array}{c}\text { RC ajusté (modèle 1) } \\
\text { (IC à 95\%) }\end{array}$} & \multicolumn{2}{|c|}{$\begin{array}{l}\text { RC ajusté (modèle 2) } \\
\text { (IC à } 95 \%)\end{array}$} \\
\hline & $\begin{array}{c}\text { Témoins } \\
(n=2 \text { 054) }\end{array}$ & $\begin{array}{l}\text { Prédiabétiques }^{\mathrm{a}} \\
\quad(\mathbf{n}=\mathbf{8 3 1})\end{array}$ & $\begin{array}{l}\text { Diabétiques }^{b} \\
(n=225)\end{array}$ & Prédiabétiques $^{\mathbf{a}}$ & Diabétiques $^{b}$ & Prédiabétiques $^{\mathrm{a}}$ & Diabétiques $^{\text {b }}$ & Prédiabétiques $^{\mathrm{a}}$ & Diabétiques $^{\mathrm{b}}$ \\
\hline Moins de 5,71 & 554 & 171 & 46 & $\begin{array}{c}1,00 \\
\text { (référence) }\end{array}$ & $\begin{array}{c}1,00 \\
\text { (référence) }\end{array}$ & $\begin{array}{c}1,00 \\
\text { (référence) }\end{array}$ & $\begin{array}{c}1,00 \\
\text { (référence) }\end{array}$ & $\begin{array}{c}1,00 \\
\text { (référence) }\end{array}$ & $\begin{array}{c}1,00 \\
\text { (référence) }\end{array}$ \\
\hline 5,71 à 11,20 & 520 & 197 & 54 & $\begin{array}{c}1,14 \\
(0,86 \text { à } 1,52)\end{array}$ & $\begin{array}{c}1,44 \\
(1,08 \text { à } 1,92)\end{array}$ & $\begin{array}{c}1,38 \\
(0,87 \text { à } 2,21)\end{array}$ & $\begin{array}{c}1,06 \\
(0,60 \text { à } 1,87)\end{array}$ & $\begin{array}{c}1,37 \\
(0,88 \text { à } 2,17)\end{array}$ & $\begin{array}{c}1,20 \\
(0,70 \text { à } 2,05)\end{array}$ \\
\hline 11,21 à 22,98 & 530 & 192 & 64 & $\begin{array}{c}1,28 \\
(0,92 \text { à } 1,62)\end{array}$ & $\begin{array}{c}1,65 \\
(1,07 \text { à } 2,54)\end{array}$ & $\begin{array}{c}1,46 \\
(0,92 \text { à } 2,32)\end{array}$ & $\begin{array}{c}1,31 \\
(0,63 \text { à } 2,74)\end{array}$ & $\begin{array}{c}1,46 \\
(0,92 \text { à } 2,35)\end{array}$ & $\begin{array}{c}1,55 \\
(0,83 \text { à } 2,90)\end{array}$ \\
\hline 22,99 et plus & 450 & 271 & 61 & $\begin{array}{c}1,48 \\
(1,18 \text { à } 2,50)\end{array}$ & $\begin{array}{c}1,92 \\
(1,11 \text { à } 3,33)\end{array}$ & $\begin{array}{c}2,04 \\
(1,03 \text { à } 4,05)\end{array}$ & $\begin{array}{c}1,54 \\
(0,74 \text { à } 3,18)\end{array}$ & $\begin{array}{c}2,14 \\
(1,02 \text { à } 4,07)\end{array}$ & $\begin{array}{c}1,81 \\
(1,12 \text { à } 2,95)\end{array}$ \\
\hline \multicolumn{4}{|c|}{$p$ pour la tendance } & 0,015 & 0,019 & 0,042 & 0,246 & 0,043 & 0,017 \\
\hline
\end{tabular}

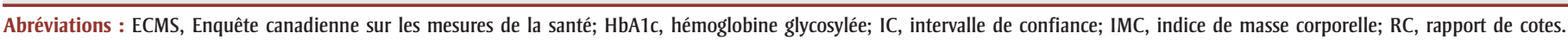

a Glycémie à jeun de 100 à 125 mg/dl (5,6 à 6,9 mmol/l) ou HbA1c de 5,7 \% à 6,4 \%.

b Glycémie à jeun de $126 \mathrm{mg} / \mathrm{dl}$ ou plus, HbA1c de 6,5\% ou plus ou autodéclaration de prise de médicaments ou de diagnostic posé par un professionnel de la santé.

c Arsenic urinaire non corrigé pour tenir compte de la créatinine urinaire.

d Il y avait des données manquantes pour 41 participants.

e Modèle 1 ajusté pour tenir compte de la créatinine urinaire, de l'âge, du sexe, de la consommation d'alcool, du tabagisme, du niveau d'éducation, de l'IMC et de l'hypertension.

${ }^{\mathrm{f}}$ Modèle 2 ajusté pour tenir compte des mêmes variables que le modèle 1 ainsi que de la consommation de produits de la mer. 
TABLEAU 5

Analyse de régression logistique ordinale multivariée comparant les participants prédiabètiques ${ }^{\mathrm{a}}$ et diabètiques ${ }^{\mathrm{b}}$ en fonction des quartiles de concentration urinaire d'arsenic, cycle 1 de l'ECMS, 2007 à 2009

\begin{tabular}{|c|c|c|c|c|c|c|}
\hline \multirow[t]{2}{*}{ Arsenic urinaire, $\mu \mathrm{g} / \mathrm{l}^{\mathrm{C}}$} & \multicolumn{3}{|c|}{ Nombre de participants ${ }^{d}$} & \multicolumn{3}{|c|}{ RC (IC à 95\%) } \\
\hline & $\begin{array}{c}\text { Témoins } \\
(\mathrm{n}=22054)\end{array}$ & $\begin{array}{l}\text { Prédiabétiques }^{\mathrm{a}} \\
\quad(\mathbf{n}=831)\end{array}$ & $\begin{array}{c}\text { Diabétiques }^{\mathbf{b}} \\
(\mathrm{n}=225)\end{array}$ & RC brut & 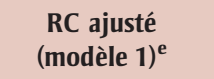 & $\begin{array}{l}\text { RC ajusté } \\
{\text { (modèle } 2)^{\mathrm{f}}}^{\text {Red }}\end{array}$ \\
\hline 5,71 à 11,20 & 520 & 197 & 54 & $1,20(0,98$ à 1,47$)$ & $1,35$ (0,95 à 1,79$)$ & $1,35(0,97$ à 1,82$)$ \\
\hline 11,21 à 22,98 & 530 & 192 & 64 & $1,20(0,88$ à 1,64$)$ & $1,39(1,01$ à 2,00$)$ & $1,41(1,02$ à 2,04$)$ \\
\hline 22,99 et plus & 450 & 271 & 61 & $1,56(1,00$ à 2,44$)$ & $1,85(1,11$ à 3,13$)$ & $1,89(1,12$ à 3,13$)$ \\
\hline
\end{tabular}

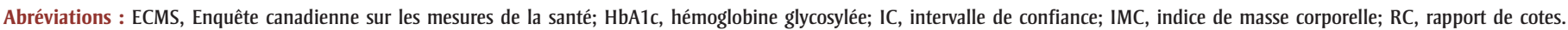

a Glycémie à jeun de 100 à 125 mg/dl (5,6 à 6,9 mmol/l) ou HbA1c : 5,7 \% à 6,4 \%.

b Glycémie à jeun de $126 \mathrm{mg} / \mathrm{dl}$ ou plus, HbA1c de $6,5 \%$ ou plus ou autodéclaration de prise de médicaments ou de diagnostic posé par un professionnel de la santé.

c Arsenic urinaire non corrigé pour tenir compte de la créatinine urinaire.

d Il y avait des données manquantes pour 41 participants.

e Modèle 1 ajusté pour tenir compte de la créatinine urinaire, de l'âge, du sexe, de la consommation d'alcool, du tabagisme, du niveau d'éducation, de l'IMC et de l'hypertension.

${ }^{\mathrm{f}}$ Modèle 2 ajusté pour tenir compte des mêmes variables que le modèle 1 ainsi que de la consommation de produits de la mer.

la concentration urinaire d'arsenic total étaient comparés aux participants du $20^{\mathrm{e}}$ percentile $(7,4 \mu \mathrm{g} / \mathrm{l}$ par rapport à $1,6 \mu \mathrm{g} / \mathrm{l})$. Ces chercheurs ont également signalé une association positive entre la concentration d'arsenic et l'HbAlc après ajustement pour les biomarqueurs de la consommation de produits de la mer (arsénobétaïne et mercure urinaires), même si l'association n'était pas statistiquement significative ${ }^{23}$.
Rhee et collab. ${ }^{26}$ ont analysé les données de l'étude transversale coréenne KNHANES (2008 à 2009) et ont noté que le RC du diabète sucré pour l'ensemble des participants était de 1,56 (IC à $95 \%: 1,03$ à 2,36 ) au sein du quartile des concentrations urinaires d'arsenic les plus élevées après ajustement pour le taux sérique de mercure comme indicateur de consommation de produits de la mer.
Les études expérimentales chez les animaux portant sur l'arsenic et le diabète sont considérées comme non concluantes en raison de leurs problèmes méthodologiques ${ }^{14}$. Des études in vitro ou mécanistiques évoquent l'existence de plusieurs voies par lesquelles l'arsenic pourrait influencer le fonctionnement des cellules $\beta$ du pancréas et la sensibilité à l'insuline, notamment le stress

\section{TABLEAU 6}

Rapport de cotes de l'hémoglobine glycosylée ${ }^{a}$ en fonction de la concentration urinaire d'arsenic chez les diabétiques traités et non traités, cycle 1 de l'ECMS, 2007 à 2009

\begin{tabular}{|c|c|c|c|c|c|c|c|c|}
\hline \multirow{3}{*}{$\begin{array}{l}\text { Arsenic } \\
\text { urinaire } \\
\left(\mu \mathrm{g} / \mathrm{l}^{\mathrm{b}}\right.\end{array}$} & \multirow{2}{*}{\multicolumn{2}{|c|}{ Nombre de participants (n) }} & \multicolumn{6}{|c|}{ RC (IC à 95\%) } \\
\hline & & & \multicolumn{2}{|c|}{ RC brut } & \multicolumn{2}{|c|}{ RC ajusté (modèle 1$)^{c}$} & \multicolumn{2}{|c|}{ RC ajusté (modèle 2) ${ }^{d}$} \\
\hline & $\begin{array}{c}\text { Diabète } \\
\text { traitée }^{\mathbf{e}} \\
(\mathbf{n}=129)\end{array}$ & $\begin{array}{c}\text { Diabète non } \\
\text { traitéf }^{\text {f }} \\
(\mathbf{n}=96)\end{array}$ & $\begin{array}{l}\text { Diabète } \\
\text { traité }\end{array}$ & $\begin{array}{c}\text { Diabète non } \\
\text { traité }\end{array}$ & $\begin{array}{l}\text { Diabète } \\
\text { traité }\end{array}$ & $\begin{array}{c}\text { Diabète non } \\
\text { traité }\end{array}$ & $\begin{array}{l}\text { Diabète } \\
\text { traité }\end{array}$ & $\begin{array}{c}\text { Diabète non } \\
\text { traité }\end{array}$ \\
\hline Moins de 5,71 & 30 & 22 & $\begin{array}{c}1 \\
\text { (référence) }\end{array}$ & $\begin{array}{c}1 \\
\text { (référence) }\end{array}$ & $\begin{array}{c}1 \\
\text { (référence) }\end{array}$ & $\begin{array}{c}1 \\
\text { (référence) }\end{array}$ & $\begin{array}{c}1 \\
\text { (référence) }\end{array}$ & $\begin{array}{c}1 \\
\text { (référence) }\end{array}$ \\
\hline 11,21 à 22,98 & 36 & 27 & $\begin{array}{c}0,94 \\
(0,58 \text { à } 1,51)\end{array}$ & $\begin{array}{c}1,21 \\
(0,89 \text { à } 1,65)\end{array}$ & $\begin{array}{c}0,85 \\
(0,46 \text { à } 1,59)\end{array}$ & $\begin{array}{c}1,72 \\
(1,13 \text { à } 2,57)\end{array}$ & $\begin{array}{c}0,80 \\
(0,48 \text { à } 1,34)\end{array}$ & $\begin{array}{c}1,74 \\
(1,18 \text { à } 2,59)\end{array}$ \\
\hline 22,99 et plus & 29 & 21 & $\begin{array}{c}1,11 \\
(0,59 \text { à } 2,04)\end{array}$ & $\begin{array}{c}1,74 \\
(1,06 \text { à } 2,89)\end{array}$ & $\begin{array}{c}0,87 \\
(0,52 \text { à } 1,46)\end{array}$ & $\begin{array}{c}2,84 \\
(1,62 \text { à } 4,98)\end{array}$ & $\begin{array}{c}0,85 \\
(0,55 \text { à } 1,32)\end{array}$ & $\begin{array}{c}2,89 \\
(1,65 \text { à } 5,08)\end{array}$ \\
\hline \multicolumn{3}{|c|}{$p$ pour la tendance } & 0,7444 & 0,005 & 0,6122 & 0,001 & 0,7538 & 0,001 \\
\hline
\end{tabular}

Abréviations : ECMS, Enquête canadienne sur les mesures de la santé; HbA1c, hémoglobine glycosylée; IC, intervalle de confiance; IMC, indice de masse corporelle; RC, rapport de cotes.

a 3 catégories d'HbA1c : inférieure à 5,7 \%, entre 5,7 \% et 6,4 \% et 6,5\% ou plus.

${ }^{\mathrm{b}}$ Arsenic urinaire non corrigé pour tenir compte de la créatinine urinaire.

c Ajusté pour tenir compte de la créatinine urinaire, de l'âge, du sexe, de la consommation d'alcool, du tabagisme, du niveau d'éducation, de l'IMC et de l'hypertension.

${ }^{\mathrm{d}}$ Ajusté pour tenir compte des mêmes variables que le modèle 1 ainsi que de la consommation de produits de la mer.

${ }^{\mathrm{e}}$ Tous les participants atteints de diabète qui prennent de l'insuline ou un hypoglycémiant oral.

${ }^{f}$ Tous les participants atteints de diabète qui ne prennent pas d'insuline ou d'hypoglycémiant oral. 
oxydatif et les effets sur l'absorption et le transport du glucose, la néoglucogenèse, la différenciation des adipocytes et la signalisation calcique ${ }^{47-50}$.

L’arsenic urinaire est généralement considéré comme l'indicateur le plus fiable de l'exposition récente à l'arsenic et il sert de principal biomarqueur de l'exposition ${ }^{51}$. L'arsenic a tendance à ne pas s'accumuler dans l'organisme : il est en effet facilement excrété par les reins ${ }^{52}$. Le profil urinaire des métabolites de l'arsenic inorganique a été utilisé dans certaines études épidémiologiques pour estimer l'exposition à l'arsenic inorganique ${ }^{14,53}$, mais ces données n'étaient pas accessibles pour le cycle 1 de l'ECMS.

L'exclusion des participants qui ont signalé une forte consommation d'aliments d'origine marine ainsi que l'ajustement des modèles en fonction des autres catégories de consommation de poisson et de fruits de mer nous ont indirectement permis de limiter la contribution des composés arsenicaux organiques de faible toxicité d'origine marine à l'arsenic total urinaire en vue d'isoler l'influence des concentrations d'arsenic inorganique. Longnecker ${ }^{54}$, dans un commentaire intitulé «On confounded fishy results regarding arsenic and diabetes », a reconnu les avantages du dosage de l'arsenic total urinaire ajusté pour les marqueurs de la consommation d'aliments d'origine marine comme indicateur de l'exposition à l'arsenic inorganique dans une population faiblement exposé $^{23}$. Cette position a toutefois été remise en question par Steinmaus et collab. ${ }^{20}$, qui n'ont noté aucune association entre le risque de diabète et l'exposition à l'arsenic inorganique en se fondant sur les métabolites inorganiques et méthylés.

Étant donné que l'eau potable est une source importante d'exposition à l'arsenic, nous avons examiné les sources d'eau potable utilisées par les participants de l'étude et nous n'avons relevé aucune association entre elles et l'état diabétique. Ce résultat pourrait être dû à la classification sommaire de l'exposition ou à la faible teneur en arsenic de l'eau potable canadienne. Le sélénium peut réduire la toxicité des espèces d'arsenic par la formation d'un complexe arsenicsélénium ${ }^{45}$ mais aucune interaction entre le sélénium et l'arsenic n’a été observée.

\section{Points forts et limites}

Comme point fort de notre étude, soulignons qu'il s'agit d'une étude en population réalisée sur un vaste échantillon d'adultes diabétiques ou prédiabétiques en fonction des critères objectifs proposés par l'ADA et l'OMS ${ }^{34,35}$. En outre, l'analyse de l'HbA1c a non seulement servi à évaluer le diabète (en l'absence d'autres critères), mais aussi à évaluer l'efficacité de la prise en charge de la glycémie. En outre, nous avons tenu compte des critères de définition du prédiabète et nous avons eu recours, dans le cadre des analyses de l'arsenic urinaire, à des procédures de laboratoire rigoureuses ayant un faible seuil de détection.

De plus, nous avons tenu compte des facteurs de confusion potentiels pertinents (facteurs de risque du diabète et indicateurs de la consommation de produits de la mer) dans notre analyse et nous avons effectué un ajustement pour la concentration urinaire de créatinine afin de tenir compte de la dilution de l'urine ${ }^{55}$.

Comme notre étude était transversale, nous n'avons pas pu établir d'association temporelle entre l'arsenic urinaire et le diabète de type 2 . L'arsenic urinaire a une demi-vie d'environ trois jours, ce qui en fait un biomarqueur uniquement pour l'exposition à court terme. Il est donc difficile de détecter les expositions antérieures qui pourraient être plus pertinentes à la pathogenèse du DT2 $2^{56}$. En outre, l'évaluation de l'exposition dans notre étude étant basée sur la concentration urinaire d'arsenic dosée dans un seul échantillon d'urine ponctuel, elle ne reflétait l'exposition qu'à un moment précis. Comme nous l'avons expliqué plus haut, nous n'avons pas quantifié les espèces d'arsenic dans l'urine, ce qui fait que nous n'avons pas pu faire d'analyse en fonction de la concentration d'arsenic inorganique ou d’arsenic organique méthylé. Nous avons plutôt ajusté la concentration d'arsenic total en fonction de la consommation de produits de la mer, en l'occurrence la principale source d'arsenic organique, conformément aux recommandations $^{23,52}$. Toutefois, comme la consommation d'aliments d'origine marine a été mesurée à l'aide d'un questionnaire sur la fréquence de consommation des aliments, l'information est sujette à des erreurs de rappel. Un biais lié aux erreurs de classification pourrait également survenir en raison de l'attribution erronée des diagnostics de DT2 : étant donné que les dossiers médicaux n’ont pas été examinés, il peut y avoir eu des erreurs liées à l'autodéclaration du diagnostic, de l'utilisation d'insuline ou de la prise d'hypoglycémiant oral. Toutefois, ce problème n'a pas semblé influencer significativement la validité des résultats primaires, car la relation positive entre l'exposition à l'arsenic mesurée dans l'urine et le DT2 a été conservée après une analyse de la sensibilité du critère biologique uniquement (HbA1c ou glycémie à jeun) chez les patients non traités. Il y avait également un taux de non-réponse important parmi les participants admissibles, ce qui pourrait avoir entraîné un biais de sélection. Toutefois, notre analyse ayant recours au score de propension semble démontrer que ce problème serait, au pire, mineur. Néanmoins, nous reconnaissons qu'une confusion résiduelle ne peut être entièrement exclue.

\section{Conclusion}

Nous avons examiné l'association entre la concentration urinaire d'arsenic total et l'état diabétique dans une population d'adultes canadiens légèrement à modérément exposés à l'arsenic par le biais de l'eau potable. À l'aide de plusieurs approches reconnues pour réduire la mauvaise classification potentielle de l'exposition due à la présence d'arsenic organique, nous avons noté une association entre l'exposition à l'arsenic total mesurée par la concentration urinaire et le DT2 dans la population étudiée. Toutefois, en raison des limites liées à la conception transversale et à l'absence d'évaluation à long terme de l'exposition à l'arsenic, nous recommandons la réalisation d'autres études prospectives avec une meilleure évaluation de l'exposition à l'arsenic. L’analyse des données récentes du cycle 2 de l'ECMS, où les données sur l'arsenic urinaire sont différenciées par sortes, pourrait également être utile. 


\section{Remerciements}

Nous remercions Statistique Canada de nous avoir fourni les données de l'Enquête canadienne sur les mesures de la santé (cycle 1). Nous remercions également Chris Le, professeur à l'Université de l'Alberta, qui était le chercheur principal titulaire de la bourse initialement attribuée pour cette étude.

Nous soulignons également la contribution et le soutien de Catherine Gonthier, ancienne coordonnatrice de la recherche au Centre de recherche du Centre hospitalier universitaire de Québec (CHUQ).

Nous avons reçu un soutien financier du Réseau canadien de l'eau (Programme des réseaux de centres d'excellence du Canada).

Les auteurs déclarent n'avoir aucun conflit d'intérêts.

\section{Références}

1. Environnement Canada, Santé Canada. L'arsenic et ses composés. Loi canadienne sur la protection de l'environnement. Introduction [Internet]. Ottawa (Ont.) : Santé Canada; [modifié le 19 avril 2007; consultation le 26 mars 2013]. Consultable en ligne à la page; http://www.hc-sc.gc.ca/ ewh-semt/pubs/contaminants/psl1-lsp1/ arsenic_comp/index-fra.php.

2. Santé Canada. Recommandations pour la qualité de l'eau potable au Canada : document technique : l'arsenic [Internet]. Ottawa (Ont.) : Santé Canada; 2006 [consultation le 26 mars 2014]. Consultable en ligne à la page; http://www.hc-sc.gc.ca/ ewh-semt/pubs/water-eau/arsenic/indexfra.php.

3. Borak J, Hosgood HD. Seafood arsenic: implications for human risk assessment. Regul Toxicol Pharmacol. 2007;47(2): 204-212.

4. Munoz E, Palmero S. Analysis and speciation of arsenic by stripping potentiometry: a review. Talanta. 2005;65(3):613-620.

5. Vahter M, Concha G. Role of metabolism in arsenic toxicity. Pharmacol Toxicol. 2001; 89(1):1-5.
6. Subcommittee on Arsenic in Drinking Water; Committee on Toxicology; Board on Environmental Studies and Toxicology; Commission on Life Sciences; National Research Council. Arsenic in drinking water. Washington (DC) : National Academy Press; 1999.

7. Tam GK, Charbonneau SM, Bryce F, Pomroy C, Sandi E. Metabolism of inorganic arsenic (74As) in humans following oral ingestion. Toxicol Appl Pharmacol. 1979;50(2):319-322.

8. Berry JP, Galle P. Selenium-arsenic interaction in renal cells : role of lysosomes electron microprobe study. J Submicrsc Cytol Pathol. 1994;26(2):203-210.

9. Chen Y, Parvez F, Gamble M, Islam T, Ahmed A, Argos $\mathrm{M}$ et collab. Arsenic exposure at low-to-moderate levels and skin lesions, arsenic metabolism, neurological functions, and biomarkers for respiratory and cardiovascular diseases : review of recent findings from the Health Effects of Arsenic Longitudinal Study (HEALS) in Bangladesh. Toxicol Appl Pharmacol. 2009;239(2):184-192.

10. Saha KC. Diagnosis of arsenicosis. J Environ Sci Health A Tox Hazard Subst Environ Eng. 2003;38(1):255-272.

11. Jones MR, Tellez-Plaza M, Sharrett AR, Guallar E, Navas-Acien A. Urine arsenic and hypertension in US adults: the 2003-2008 National Health and Nutrition Examination Survey. Epidemiology. 2011;22(2):153-161.

12. Kwok RK, Mendola P, Liu ZY, Savitz DA, Heiss G, Ling HL et collab. Drinking water arsenic exposure and blood pressure in healthy women of reproductive age in Inner Mongolia, China. Toxicol Appl Pharmacol. 2007;222(3):337-343.

13. Mukherjee SC, Rahman MM, Chowdhury UK et collab. Neuropathy in arsenic toxicity from groundwater arsenic contamination in West Bengal, India. J Environ Sci Health A Tox Hazard Subst Environ Eng. 2003;38(1):165-183.

14. Maull EA, Ahsan H, Edwards J et collab. Evaluation of the association between arsenic and diabetes : a National Toxicology Program workshop review. Environ Health Perspect. 2012;120(12):1658-1670.

15. Lopez AD, Mathers CD, Ezzati M, Jamison DT. Measuring the global burden of disease and risk factors, 1990-2001. Dans : Lopez AD, Mathers CD, Ezzati M, Jamison DT (dir.), Global burden of disease and risk factors. Washington (DC) : World Bank; 2006.
16. Kapur A, Harries AD. The double burden of diabetes and tuberculosis - public health implications. Diabetes Res Clin Pract. 2013;101(1):10-19.

17. Pelletier C, Dai S, Roberts KC, Bienek A, Onysko J, Pelletier L. Le diabète au Canada : perspective de santé publique sur les faits et chiffres. Maladies chroniques et blessures au Canada. 2012;33(1):53-54.

18. Abernathy CO, Thomas DJ, Calderon RL. Health effects and risk assessment of arsenic. J Nutr. 2003. 133(5 Suppl 1)1536S$1538 \mathrm{~S}$.

19. Chen Y, Ahsan H, Slavkovich V et collab. No association between arsenic exposure from drinking water and diabetes mellitus : a cross-sectional study in Bangladesh. Environ Health Perspect. 2010;118(9):12991305.

20. Steinmaus C, Yuan Y, Liaw J, Smith AH. Low-level population exposure to inorganic arsenic in the United States and diabetes mellitus: a reanalysis. Epidemiology. 2009;20(6):807-815.

21. Gribble MO, Howard BV, Umans JG et collab. Arsenic exposure, diabetes prevalence, and diabetes control in the Strong Heart Study. Am J Epidemiol. 2012;176(10): 865-874.

22. Del Razo LM, García-Vargas GG, Valenzuela OL et collab. Exposure to arsenic in drinking water is associated with increased prevalence of diabetes : a cross-sectional study in the Zimapan and Lagunera regions in Mexico. Environ Health. 2011;10:73.

23. Navas-Acien A, Silbergeld EK, Pastor-Barriuso R, Guallar E. Rejoinder : Arsenic exposure and prevalence of type 2 diabetes : updated findings from the National Health Nutrition and Examination Survey, 2003-2006. Epidemiology. 2009;20(6):816-820.

24. Coronado-Gonzalez JA, Del Razo LM, García-Vargas G, Sanmiguel-Salazar F, Escobedo-de la Peña J. Inorganic arsenic exposure and type 2 diabetes mellitus in Mexico. Environ Res. 2007;104(3): 383-389.

25. Wang SL, Chang FH, Liou SH, Wang HJ, Li WF, Hsieh DP. Inorganic arsenic exposure and its relation to metabolic syndrome in an industrial area of Taiwan. Environ Int. 2007;33(6):805-11. 
26. Rhee SY, Hwang YC, Woo JT, Chin SO, Chon $\mathrm{S}$, Kim YS. Arsenic exposure and prevalence of diabetes mellitus in Korean adults. J Korean Med Sci. 2013;28(6):861-868.

27. Kim NH, Mason CC, Nelson RG et collab. Arsenic exposure and incidence of type 2 diabetes in Southwestern American Indians. Am J Epidemiol. 2013;177(9):962-969.

28. Giroux S. Enquête canadienne sur les mesures de la santé : aperçu de la stratégie d'échantillonnage. Rapports sur la santé. 2007;18Suppl:31-36.

29. Tremblay M, Wolfson M, Connor Gorber S. Enquête canadienne sur les mesures de la santé : raison d'être, contexte et aperçu. Rapports sur la santé. 2007;18Suppl:7-20.

30. Schneider AL, Lazo M, Ndumele CE et collab. Liver enzymes, race, gender and diabetes risk: the Atherosclerosis Risk in Communities (ARIC) Study. Diabet Med. 2013;30(8):926-33.

31. Valcarcel M. Lucena R. Synergistic relationships between analytical chemistry and written standards. Anal Chim Acta. 2010;788:1-7.

32. Institut national de santé publique du Québec. Méthode d'analyse pour doser les métaux et autres éléments dans l'urine par spectrométrie de masse à plasma d'argon induit (ICP-MS), DRC II (M-571), version condensée et pour certains éléments de sélection. Québec (QC) : Laboratoire de toxicologie; 2009.

33. Jaffe M. Hoppe-Seyler's Z. Physiol Chem. 1886;10:391-400.

34. American Diabetes Association. Diagnosis and classification of diabetes mellitus. Diabetes Care. 2013;36 Suppl 1:S67-S74.

35. International Expert Committee. International Expert Committee report on the role of the A1C assay in the diagnosis of diabetes. Diabetes Care. 2009;32(7):1327-1334.

36. Ortho-Clinical Diagnostics, $\mathrm{n}$ de publication MP2-8_FR (version 4.0), Plaques GLU VITROS Chemistry Products.

37. Ortho-Clinical Diagnostics, $\mathrm{n}$ de publication J23413_FR (version 2.0), Réactifs VITROS Chemistry Products A1C.

38. Institut national de santé publique du Québec. Méthode d'analyse pour doser la créatinine dans l'urine par (C-530), version condensée. Québec (QC) : Laboratoire de toxicologie; 2009.
39. Campbell NR, Joffres MR, McKay DW. Hypertension surveillance in Canada: minimum standards for assessing blood pressure in surveys. Can J Public Health. 2005;96(3):217-220.

40. Rao JN, Wu CF, Yue K. Quelques travaux récents sur les méthodes de rééchantillonnage applicables aux enquêtes complexes. Techniques. 1992;18(2):225-234. d'enquête (Statistique Canada, n 12-001 au catalogue).

41. Enquête canadienne sur les mesures de la santé (ECMS). Guide de l'utilisateur des données : cycle 1 [Internet]. Ottawa (Ont.) : Statistique Canada; 2011 [modifié le 4 avril 2014; consultation le 10 juillet 2014]. Consultable en ligne à l'adresse; http://www23 . statcan.gc.ca/imdb-bmdi/pub/document/ 5071_D2_T1_V1-fra.htm.

42. Cole SR, Chu H, Nie L, Schisterman EF. Estimating the odds ratio when exposure has a limit of detection. Int J Epidemiol. 2009;38(6):1674-1680.

43. Vahter M, Bjorkman L, Goessler W. Concentrations of biomarkers in spot urine samples need adjustment for variation in dilutionComment on: "Distribution of urinary selenium and arsenic among pregnant women exposed to arsenic in drinking water" [Environ Res. 2006. 100(1) : 115-122]. Environ Res. 2007;104(2):312-313, discussion 314.

44. Koenig RJ, Peterson CM, Jones RL, Saudek C, Lehrman M, Cerami A. Correlation of glucose regulation and hemoglobin AIc in diabetes mellitus. N Engl J Med. 1976;295(8):417-420.

45. Alp O, Zhang Y Merino EJ, Caruso JA Selenium effects on arsenic cytotoxicity and protein phosphorylation in human kidney cells using chip-based nanoLC-MS/MS Metallomics. 2011;3(5):482-490.

46. Austin PC. An introduction to propensity score methods for reducing the effects of confounding in observational studies. Multivariate Behav Res. 2011;46:399-424.

47. Diaz-Villasenor A, Burns AL, Salazar AM et collab. Arsenite reduces insulin secretion in rat pancreatic b-cells by decreasing the calcium-dependent calpain-10 proteolysis of SNAP-25. Toxicol Appl Pharmacol. 2008; 231(3):291-299.

48. Druwe IL, Vaillancourt RR. Influence of arsenate and arsenite on signal transduction pathways: an update. Arch Toxicol. 2010; 84(8):585-596.
49. Tseng $\mathrm{CH}$. The potential biological mechanisms of arsenic-induced diabetes mellitus. Toxicol Appl Pharmacol. 2004; 197(2):67-83.

50. Fu J, Woods CG, Yehuda-Shnaidman E et collab. Low-level arsenic impairs glucosestimulated insulin secretion in pancreatic beta-cells: involvement of cellular adaptive response to oxidative stress. Environ Health Perspect. 2010;118:864-870.

51. Calderon RL, Hudgens E, Le XC, Schreinemachers D, Thomas DJ. Excretion of arsenic in urine as a function of exposure to arsenic in drinking water. Environ Health Perspect. 1999;107(8):663-667.

52. Vahter M, Lind B. Concentrations of arsenic in urine of the general population in Sweden. Sci Total Environ. 1986;54:1-12.

53. Valenzuela OL, Borja-Aburto VH, GarciaVargas GG et collab. Urinary trivalent methylated arsenic species in a population chronically exposed to inorganic arsenic. Environ Health Perspect, 2005;113(3):250254.

54. Longnecker MP. On confounded fishy results regarding arsenic and diabetes. Epidemiology. 2009;20(6):821-823.

55. Basu A, Mitra S, Chung J et collab. Creatinine, diet, micronutrients, and arsenic methylation in West Bengal, India. Environ Health Perspect. 2011;119(9):1308-1313.

56. Chen YC, Amarasiriwardena CJ, Hsueh YM, Christiani DC. Stability of arsenic species and insoluble arsenic in human urine. Cancer Epidemiol Biomarkers Prev. 2002; 11(11):1427-1433. 